\title{
Effect of chinese herbal medicine on female infertility
}

\begin{abstract}
Infertility is the inability of a sexually active, non-contracepting couple to achieve pregnancy in one year. In recent years, more couples are looking to alternative and complementary medicine (CAM) to achieve a satisfactory result, although conventional treatment possesses many effective therapies to treat infertility. Chinese Herbal Medicine (CHM) can be a very effective treatment. Through combining multiple therapies from within this traditional medical system, such as CHM and acupuncture together, CHM, otherwise known as TCM, can enhance the natural pregnancy rate two-fold, which is remarkably higher than through routine hormonal treatment. Evidence has also shown that CHM can increase the success rate of Assistant Reproductive Techniques (ART) from 33\% to 60\%. Authors here have reviewed CHM research papers from China, Asia, Europe and America which explain how to treat infertility using CHM based on clinical experience, how CHM supports ART, and also pre-clinical research which seeks to prove CHM's effectiveness, and demonstrate its mechanism of action from laboratory and animal tests. The key techniques of CHM are either using a tried-and-tested formula composed of a mixture of several herbs according to classical theory or clinical experience of a famous doctor, or by using a combination of multiple traditional therapies together. Some researchers advocate integrating CHM with surgery to encourage post-operative healing and promote early patient recovery.
\end{abstract}

Keywords: Infertility; Chinese Herbal Medicine; TCM
Volume 8 Issue I - 2017

\author{
Dan Jiang,' Lily Li² \\ 'Hallam Institute of TCM, UK \\ ${ }^{2} \mathrm{St}$ Marys Hospital Paddington, UK
}

Correspondence: Ms Dan Jiang, Fellow of BAcC, Fellow of ATCM, TCM Consultant (be awarded by WFCMS), Principal of Hallam Institute of TCM UK, Vice Principal of Asante Academy of Chinese Medicine UK,Visiting Professor of Beijing University of Chinese Medicine in China. Email djiang52@hotmail.com

Received: August 28, 2017 | Published: September 27, 2017

\section{Introduction}

Infertility is defined as an absence of successful pregnancy in a couple where the woman is 35 years and younger, with at least one year of regular intercourses without using birth control methods. Fertility decreases with age. ${ }^{1}$ Estimated infertility from 782 couples recruited from seven European centres is 8\% for women aged 19-26 years, $13-14 \%$ for women aged $27-34$ years, and $18 \%$ for women aged 35-39 years. ${ }^{2}$ women aged 15-44 in the USA with impaired fertility is $12.3 \%{ }^{3}$ Common reasons causing female infertility are tubal blockage, pelvic inflammatory disease caused by infections such as tuberculosis, uterine problems, previous tubal ligation; endometriosis, and advanced maternal age. ${ }^{4}$ Up to $20 \%$ of infertile couples have unexplained infertility. In these cases, abnormalities are likely to be present but not detected by current methods, or only functional disorders are apparent. ${ }^{5}$

Medical treatment of infertility generally involves the use of fertility medication[follicle-stimulating hormone (FSH), human chorionic gonadotropin (hCG), gonadotropin-releasing hormone $(\mathrm{GnRH})$ analogues], ovarian stimulating medication [clomiphene citrate, human menopausal gonadotropin (hMG), Letrozole). ${ }^{6}$ surgery (tuboplasty, salpingectomy, oophorectomy, unilateral or bilateral salpingo-oophorectomy (USO), ${ }^{7,8}$ or assisted reproductive technology (ART) techniques such as in vitro fertilization (IVF) and related techniques (ICSI, ZIFT, GIFT). ${ }^{9}$

These therapies can treat some causes of infertility but results are not always satisfactory. Although the overall live birth rate per embryo transfer has increased from $19.2 \%$ in 2002 to $23.3 \%$ in 2013 (21.9$24.3 \%$ for fresh embryo transfers and $14.6-23.3 \%$ for frozen/thaw embryo transfers). ${ }^{9,10}$ the result still falls short of the expectations of the infertile woman/couple. Furthermore side effects from hormonal treatment are not without risk, such as overstimulated ovaries. ${ }^{11}$ or psychiatric disorders. ${ }^{12}$

Due to the lack of a perfectly satisfactory outcome from standard medical treatment, patients are looking to alternative therapies to improve their results. Acupuncture has been proven by many different studies from both laboratory and clinic to play an important role in supporting infertility treatment. ${ }^{13-15}$ Chinese Herbal Medicine is an integral part of Traditional Chinese Medicine, in which acupuncture, diet, exercise (Taiji)and Qigong are involved together as a whole system of health maintenance. Do they play an important role in supporting infertility treatment? As a traditional medical system, how can this system of TCM be used to give the best result or infertility treatment? In this chapter we perform a thorough review of papers.

Key words "Chinese Herbal Medicine, or TCM, and Infertility" were used in the Pubmed, Cochrane, Medline, Google Scholar, CINAHL and Wanfang databases to search for papers published between 20012016.The resultant papers were evaluated closely and summarised below. In general most of research on the mechanism of infertility treatment using $\mathrm{CHM}$ or TCM have come from research teams in China, as well as other Asian countries such as Japan. ${ }^{17,18}$ Korea. ${ }^{19}$ and Taiwan. ${ }^{20}$ However increasingly more papers are being published from Western countries such as Australia, the USA, and Europe.. Below is a summary of the findings from literature review:

\section{Pre-clinic and laboratory research:}

There are many studies which aim to elucidate the mechanism of Chinese Herbal Medicine in treating infertility in rat models of common diseases associated with infertility.

To promote endometrial receptivity in the uterus: To conceive either naturally or using an assisted pregnancy technique, a receptive uterine state is required. Poor endometrial receptivity is the commonest reason for infertility and prevents embryo implantation into the uterus. $\mathrm{CHM}$ has been found to promote a receptive uterine state.

In Y Nan's study using a mouse model,163 female pregnant kunming mice were randomly divided into six groups, comprising the A control group; B ovulation stimulation (OS) group; C OS+TCM (Chinese herbal medicine Pregnancy-supporting Recipe - the Zhuyun Recipe, Table 1) group; D embryo implantation 
dysfunction (EID) group; E, EID+TCM group; F TCM-only group. Uterine samples were collected at gestation day four and detected with immunohistochemistry and Real Time-PCR analyses. Uterine horns were excised to determine the number of pregnant mice and implantation sites on day eight post-coitus.

The study found that OS group and EID group showed a significant decrease in pregnancy rate and the expression of both endometrial leukaemia inhibitory factor (LIF) and integrin $\beta 3$ subunit during the implantation window. The OS+TCM and EID+TCM groups showed a higher pregnant rate and endometrial LIF and integrin $\beta 3$ subunit expression compared to the OS and EID groups. The number of implanted embryos in EID group was lower than in the control group, but higher in the EID+TCM group than in the EID group. Their research indicates that TCM appears to reverse the expression of endometrial LIF and integrin $\beta 3$ subunit, improves the uterine receptivity in mice, and increases pregnancy rate and embryonic implantation..$^{21}$

X Gong's team showed that Leukemia-inhibitory factor (LIF) and Angiopoietin-2 (Ang-2) are important factors in fertility. Bu Shen HuoXue Decoction (BSHXD, an established CHM recipe, Table 1) was investigated for its effect on the number of implantation sites and live births in rats. Uteri were collected on day (D) 3, 4 and 5 of pregnancy, and LIF and Ang-2 protein and mRNA expression were detected using Western blot analysis and quantitative polymerase chain reaction. On pregnancy D10, the average number of implantation sites was observed, and the number of live births from each group was recorded. The study found that BSHXD treatment markedly increased the number of live births by restoring endometrial LIF expression and the implantation capacity in the rat model. In addition, no association was identified between LIF and Ang-2 expression. The author s concluded that BSHXD may be useful in female reproduction. ${ }^{22}$

To stimulate ovulated inhibition and to treat ovary failure, and to raise hormonal levels: Premature ovarian failure and insufficient storage function of the ovaries are both common reasons for infertility. They can be a result of a variety of hormonal disorders. Established recipes of CHM have been found to effectively stimulate ovaries and raise low hormone level.

S Luo's research team used rat models where ovulation was inhibited (by hydroxyl urea), which matched Shen deficiency and dampness-accumulation patterns in TCM. They showed that Luo's established recipe (Table 1) increased ovulation, levels of FSH, LH and oestrogen, and promoted pregnancy rate when compared to the control group..$^{23}$

X Teng's team gave 60 female rats Tripterygium wilfordii tablets orally to establish a rat model of premature ovarian failure. The rats were randomly divided into a control group $(\mathrm{n}=10)$, and model group $(\mathrm{n}=50)$. The latter group were given Yulin Formula which is a classical established CHM recipe for treating Shen deficiency and blood stasis (Table 1). The test group was also sub-divided into groups of low, middle, and high dose Yulin formula. Their results showed that Yulin formula increased ovarian wet weight in rats with premature ovarian failure and increased the ovarian index. The study also showed that Yulin formula reduced FSH and LH levels, and raised oestrogen levels, They found also that Yulin formula promoted follicle growth and repair, thus improving ovarian function. ${ }^{24}$

To relax the uterus and to prevent spontaneous abortion: Luo's team designedrat models of abortion using hydroxyurea and mifepristone to mimic Kidney deficiency and Spleen deficiency respectively for observing the effect of ZY3 (CHM recipe for Pregnancy-support:Zhuyun No3, an establised formula for preventing miscarriage, Table 1) on Progesterone (P) and Estradiol (E2) and their receptors (PR and ER). They found that $\mathrm{ZY} 3$ could significant increase positive rates of $P R$ in decidua models of two types: its water abstract and drug serum can decrease the potency of contractility of the uterus in vivo, and isolated uterus smooth muscle in vitro respectively. They concluded that the major mechanism of ZY3 in reinforcing Kidney and strengthening Spleen in protecting pregnancy is to elevate target cell PR and relax uterine smooth muscle. . $^{25.26}$

To comprehensive observation to embryo embedding condition of uterus for increase a natural pregnancy and IVF's successful rates which is supported by CHM: $\mathrm{H}$ Du et al. [27] studied the effect of recipes for reinforcing Kidney and dredging Liver on strengthening endometrial receptivity to improve pregnancy rates. They found that two-thirds of pregnancy failure was caused by poor uterine receptivity. They designed a mouse model with recurrent failure of IVF-ET. After giving injection of hCG to the test and control groups of mice, they observed levels of E2, $\mathrm{LH}$, and progesterone, the thickening of the endometrium, and expression of angiogenic proteins at the endometrial surface. They found that the Bushenzhuyun Recipe (Table 1) increased hormone levels and thicked the endometrium. It also downregulated the expression of ER, PR, and up-regulated expression of VEGF, VEGFR-2, eNOS, PCNA, MMP-9, and CyclinD1, which are involved in promoting cellular growth, increasing vasculogenesis, and increasing vascular permeability. They concluded that CHM could correct uterus receptivity. ${ }^{27}$

Chinese Herbal Medicine can increase pregnancy rate in clinical research: K Ried's team performed a systematic review in 2011 looking at Chinese herbal medicine in infertility. They identified eight randomised controlled trials (RCTs), 13 cohort studies, three case series, and six case studies involving 1851 women with infertility. Meta-analysis of the RCTs suggested a 3.5 greater likelihood of achieving pregnancy with CHM therapy over a four-month period compared with Western drug (clomiphene) therapy alone. They suggested that management of female infertility with Chinese Herbal Medicine could improve pregnancy rates two-fold.$^{28}$ They also found that assessment of the quality of the menstrual cycle, integral to TCM diagnosis, appears to be fundamental to successful treatment of female infertility. ${ }^{29}$

The same group published an updated review of the same topic in 2015, where they collected data from 40 RCTs involving 4247 women with infertility into a systematic review. Meta-analysis suggested a 1.74 higher probability of achieving pregnancy with CHM therapy than with Western therapy alone. In addition the authors concluded that fertility indicators such as ovulation rates, cervical mucus score, biphasic basal body temperature, and appropriate thickness of the endometrial lining are positively influenced by CHM therapy, indicating an ameliorating physiological effect for viable pregnancy. ${ }^{30}$

To promote ovulation rate: $\mathrm{L}$ Tan. ${ }^{31}$ published a meta-analysis where 1659 participants were involved in 15 studies showing the efficacy of CHM treatment of infertility caused by anovulation as compared to clomiphene. Analysis indicated that CHM significantly increased the pregnancy rate. There were also no significant adverse effects identified from the use of CHM in this review. ${ }^{31}$

Y Huang's team divided 60 patients with anovulatory infertility into a Chinese medicine treatment group and a Western medicine control group where the groups were equally matched in their general and anovulatory condition. The treatment group was treated with LEL (an established CHM recipe, Table 1), and the control group was given clomiphene. Ovulation rates and pregnant rates were observed and compared. They found that the total effective rate in the treatment group was $96.7 \%$, which was significantly higher than that in the 
control group $(53.3 \%, \mathrm{p}<0.05)$. The abortion rate was $10.0 \%$ in the treatment group, which was significantly lower than that in the control group $(54.6 \%, \mathrm{p}<0.05)$. The symptom score decrease significantly in the treatment group after treatment $(\mathrm{P}<0.01)$, but remained unchanged

Table I The established CHM formulae mentioned in this review, and the constituent herbs.

\begin{tabular}{|c|c|c|c|c|c|}
\hline $\begin{array}{l}\text { Reference number } \\
\text { \& Author }\end{array}$ & Name of formula & Herbs in formula & Latin names of herbs & Pattern of TCM & Research form \\
\hline X Gong. ${ }^{6}$ & $\begin{array}{l}\text { Bushenhuoxue } \\
\text { Recipe (Tonifying Kid and } \\
\text { Activating Blood Formula) }\end{array}$ & $\begin{array}{l}\text { Sheng Di Huang } \\
\text { Dan Shen } \\
\text { Dang Gui } \\
\text { ChuanDuan } \\
\text { Du Zhong } \\
\text { Shan Yao } \\
\text { Mei Gui-hua } \\
\text { ChuanXiong } \\
\text { YiYi-ren }\end{array}$ & $\begin{array}{l}\text { Rehmannia Rehmanniae } \\
\text { Salviaemiltiorrhizae Bge. } \\
\text { Radix Angelica sinensis (Oliv.) } \\
\text { DipsacusAsperoides Diels., } \\
\text { Eucommiaulmoides Oliv., } \\
\text { cRhizomaDioscorea } \\
\text { FlosRosa rugosa } \\
\text { Ligusticum } \\
\text { Rhizomachuanxiong } \\
\text { Semen Coicis }\end{array}$ & $\begin{array}{l}\text { Kidney Deficiency } \\
\text { \& Blood } \\
\text { stasis }\end{array}$ & Rat Model \\
\hline S Luo. ${ }^{7}$ & Luo's recipe & $\begin{array}{l}\text { Tuisizi } \\
\text { Dangshen } \\
\text { Gouqizi } \\
\text { Bajitian } \\
\text { Shudihuang } \\
\text { Yinyanghuo } \\
\text { Dangui } \\
\text { Fuzi } \\
\text { Zhigancao }\end{array}$ & $\begin{array}{l}\text { Semen Cuscutae } \\
\text { Radix Codonopsis } \\
\text { FructusLycii Chinensis } \\
\text { Radix Morindae Officinalis } \\
\text { Radix Glutinosa Libosch } \\
\text { Herba Epimedi } \\
\text { Radix Angelicae Sinensis } \\
\text { Radix Aconiti Lateralis Preparata } \\
\text { Angelica Sinensis (Oliv.)Diels } \\
\text { Aconitum Carmichaeli Debx } \\
\text { Radix Glycyrrhizae Preparata }\end{array}$ & $\begin{array}{l}\text { Kidney deficiency } \\
\text { \& } \\
\text { Dampness } \\
\text { accumulation }\end{array}$ & Rat Model \\
\hline X Teng. ${ }^{8}$ & $\begin{array}{l}\text { Yulin Formula } \\
\text { (A classical recipe } 39 \text { ) }\end{array}$ & $\begin{array}{l}\text { Renshen, } \\
\text { Baizhu, } \\
\text { Fuling, } \\
\text { Baishao, } \\
\text { Chuanxiong, } \\
\text { Danggui, } \\
\text { Shudihuang, } \\
\text { Tusizi, } \\
\text { Duzhong, } \\
\text { Lujiaoshuang } \\
\text { Chuanjiao } \\
\text { Zhigancao }\end{array}$ & $\begin{array}{l}\text { Ranax Ginseng C.A.Mey } \\
\text { Atractylodes Macrocephala Koidz } \\
\text { Poria Cocos (Schw)Wolf } \\
\text { Paeonia Laciflora Pall } \\
\text { Ligusticum ChuanxionHort } \\
\text { Angelica Sinensis (Oliv.)Diels } \\
\text { Rehmannia Glutinosa Libosch } \\
\text { Cuscuta Chinensis Lam } \\
\text { Eucommia UlmoidesOliv } \\
\text { Zanthoxylum Bungeanum Maximl } \\
\text { Radix Glycyrrhizae Preparata }\end{array}$ & $\begin{array}{l}\text { Kidney deficiency } \\
\text { \& } \\
\text { Blood deficiency }\end{array}$ & $\begin{array}{l}\text { Rat } \\
\text { Model }\end{array}$ \\
\hline S Luo.9 & $\begin{array}{l}\text { Zhuyun Recipe No3 } \\
\text { (Promoting conceive } \\
\text { recipe) }\end{array}$ & $\begin{array}{l}\text { Tusizi, } \\
\text { Sangjisheng } \\
\text { Hunagqi, } \\
\text { Dangshen, } \\
\text { Xuduan, } \\
\text { Nvzhenzi }\end{array}$ & $\begin{array}{l}\text { CuscutaChinensis Lam } \\
\text { Taxillus Chinensis (DC.) Danser } \\
\text { Astragalus Membranaceus (Fisch) } \\
\text { Codonopsis Pilosula (Franch.) Nannf } \\
\text { Dipsacus Asperoides } \\
\text { Ligustrum Lucidum Ait. }\end{array}$ & $\begin{array}{l}\text { Kidney\& } \\
\text { Spleen deficiencies, } \\
\text { Blood stasis }\end{array}$ & \\
\hline H Du." & $\begin{array}{l}\text { Bushenzhuyun recipe } \\
\text { (Tonifying Kid \& promoting } \\
\text { conceive }\end{array}$ & $\begin{array}{l}\text { Shudihuang } \\
\text { Dangui } \\
\text { Baishao } \\
\text { Shanyao } \\
\text { Shanyurou } \\
\text { Gouqizi } \\
\text { Yinyanghuo } \\
\text { Huangqi } \\
\text { Tusizi }\end{array}$ & $\begin{array}{l}\text { Rehmannia Glutinosa Libosch } \\
\text { Angelica Sinensis (Oliv.)Diels } \\
\text { Paeonia Laciflora Pall } \\
\text { Diosacorea OppositaThunb. } \\
\text { Cornus Officinalis Sieb } \\
\text { Fructus LyciiChinensis } \\
\text { Epimedium Brevicornum Maxim. } \\
\text { Astragalus Membranaceus (Fisch } \\
\text { Cuscuta Chinensis Lam }\end{array}$ & $\begin{array}{l}\text { Kidney deficiency } \\
\& \\
\text { Liver stagnation }\end{array}$ & Rat Model \\
\hline Q Chen. ${ }^{16}$ & $\begin{array}{l}\text { Tiaojingyunyu Recipe } \\
\text { (regulating menstrual circle } \\
\text { and promoting conceive } \\
\text { formula 39) }\end{array}$ & $\begin{array}{l}\text { Dangui, } \\
\text { Chuanxiong } \\
\text { Shudihuang } \\
\text { Fuling } \\
\text { Chenpi } \\
\text { Xiangfu } \\
\text { Wuzhuyu } \\
\text { Rougui } \\
\text { Ganjiang } \\
\text { Mudanpi, } \\
\text { Yanhusuo, } \\
\text { Aiye }\end{array}$ & $\begin{array}{l}\text { Radix Angelica sinensis (Oliv.) } \\
\text { Ligusticum ChuanxionHort } \\
\text { Radix Glutinosa Libosch } \\
\text { Poria Cocos (Schw)Wolf } \\
\text { Citrus Reticulata Blanco } \\
\text { Cyperus Rotundus L. } \\
\text { Evodia Rutaecarpa (Juss.) Benth. } \\
\text { Cinnamomum Cassia Presl } \\
\text { Zingiber OfficinaleRosc. } \\
\text { Paeonia SuffruticosaAndr. } \\
\text { Cordalis Vanhusuo } \\
\text { Artemisia ArgyiLevl. }\end{array}$ & $\begin{array}{l}\text { Blood deficiency } \\
\& \text { stasis }\end{array}$ & Clinic \\
\hline
\end{tabular}

in the control group. The authors were able to conclude that LEL had good effect in treating anovulatory infertility. Q Chen and X Li's team have made similar studies with positive results. ${ }^{32-34}$ 
Table Continued...

\begin{tabular}{|c|c|c|c|c|c|}
\hline $\begin{array}{l}\text { Reference number } \\
\text { \& Author }\end{array}$ & Name of formula & Herbs in formula & Latin names of herbs & Pattern of TCM & Research form \\
\hline Y Huang. ${ }^{18}$ & Lin' erlai Recipe & $\begin{array}{l}\text { Guoqizi } \\
\text { Tusizi } \\
\text { Fupenzi } \\
\text { Wuweizi } \\
\text { Cheqianzi, } \\
\text { Shudihuang } \\
\text { Baishao } \\
\text { Danggui } \\
\text { Chuanxiong }\end{array}$ & $\begin{array}{l}\text { LyciumBarbarum L. } \\
\text { CuscutaChinensis Lam } \\
\text { RubusChingii Hu } \\
\text { Schisandra Sphenanthera } \\
\text { Plantago Asiatica L. } \\
\text { Cuscuta Chinensis Lam } \\
\text { Paeonia Laciflora Pall } \\
\text { Angelica Sinensis (Oliv.)Diels } \\
\text { Ligusticum ChuanxionHort }\end{array}$ & $\begin{array}{l}\text { Kidney \& } \\
\text { Blood deficiencies }\end{array}$ & Clinic \\
\hline $\begin{array}{l}\text { J Zhou. }{ }^{20} \mathrm{Y} \text { Jiang. }{ }^{23} \mathrm{~J} \\
\text { Guo } .^{32}\end{array}$ & $\begin{array}{l}\text { Tonifying Kid and Activating } \\
\text { blood Recipe } \\
\text { Re }\end{array}$ & $\begin{array}{l}\text { Huangqi } \\
\text { Danshen } \\
\text { Baizhu } \\
\text { Sanyao } \\
\text { Shanzhuyu } \\
\text { Nvzhenzi } \\
\text { Hanliancao } \\
\text { Xianmao } \\
\text { gYinyanghuo } \\
\text { Chuanxiong } \\
\text { Fuling } \\
\text { Honghua } \\
\text { Danggui } \\
\text { Tusizi } \\
\text { Chenpi } \\
\text { Muxiang } \\
\text { Zexie }\end{array}$ & $\begin{array}{l}\text { Astragalus Membranaceus(Fisch) } \\
\text { Salviaemiltiorrhizae Bge. } \\
\text { Atractylodes MacrocephalaKoidz } \\
\text { Diosacorea OppositaThunb. } \\
\text { Cornus Officinalis Sieb } \\
\text { Ligustrum Lucidum Ait. } \\
\text { Eclipta Prostrata L. } \\
\text { Curculigo OrchioidesGaeth } \\
\text { Epimedium Brevicornum Maxim. } \\
\text { Ligusticum Chuanxion Hort. } \\
\text { Poria Cocos (Schw.)Wolf } \\
\text { CarpesiumTinctorins L } \\
\text { Angelica Sinensis (Oliv.)Diels } \\
\text { CuscutaChinensis Lam } \\
\text { Citrus Reticulata Blanco } \\
\text { Vladimiria Souliei (Franch.) Ling } \\
\text { Alisma Orientalis (Sam) Juzep }\end{array}$ & $\begin{array}{l}\text { Kidney deficiency } \\
\& \\
\text { Blood stasis }\end{array}$ & Clinic \\
\hline F Liang. ${ }^{24} \mathrm{~F}$ Lian. ${ }^{3 \prime}$ & ErzhiTiangui Granule & $\begin{array}{l}\text { Nvzhenzi } \\
\text { Hanliancao }\end{array}$ & $\begin{array}{l}\text { Ligustrum Lucidum Ait. } \\
\text { Eclipta Prostrata L. }\end{array}$ & $\begin{array}{l}\text { Kidney Yin } \\
\text { deficiency }\end{array}$ & Clinic \\
\hline C Lu. ${ }^{25}$ & $\begin{array}{l}\text { BushenHua } \\
\text { tan Recipe (Tonifying Kid } \\
\text { and Cleansing Phlegm } \\
\text { formula ) }\end{array}$ & $\begin{array}{l}\text { Huangqi } \\
\text { Canzhu } \\
\text { Fuling } \\
\text { Yinyanghuo } \\
\text { Danshen }\end{array}$ & $\begin{array}{l}\text { Astragalus Membranaceus (Fisch) } \\
\text { Xanthium Sibiricum Part. } \\
\text { Poria Cocos (Schw.) Wolf } \\
\text { Epimedium Brevicornum Maxim. } \\
\text { Salvia MiltiorrhizaBge }\end{array}$ & $\begin{array}{l}\text { Kidney deficiency } \\
\text { \& Phlegm } \\
\text { accumulation }\end{array}$ & Clinic \\
\hline $\mathrm{YChen}^{26}$ & $\begin{array}{l}\text { Bushenantai Recipe } \\
\text { (Tonifying Kid to protect } \\
\text { embryo ) }\end{array}$ & $\begin{array}{l}\text { Baishao } \\
\text { Chuanduan } \\
\text { Sangjisheng } \\
\text { Longgu } \\
\text { Muli } \\
\text { Gancao } \\
\text { Ejiao }\end{array}$ & $\begin{array}{l}\text { Paeonia Laciflora Pall } \\
\text { Dipsacus Asperoides } \\
\text { Taxillus Chinensis (DC.) Danser } \\
\text { (Fossilised Bone) } \\
\text { (Oyster Shell) } \\
\text { Glycyrrhizae Uralensis Fisch } \\
\text { Asini Gelatinun, }\end{array}$ & $\begin{array}{l}\text { Kidney \&Spleen } \\
\text { deficiency }\end{array}$ & Clinic \\
\hline Ju. ${ }^{28}$ & $\begin{array}{l}\text { Yupingfeng Recipe (A } \\
\text { classical formula) }\end{array}$ & $\begin{array}{l}\text { Huangqi } \\
\text { Baizhu } \\
\text { Fangfeng }\end{array}$ & $\begin{array}{l}\text { Astragalus Membranaceus (Fisch) } \\
\text { Atractylodes MacrocephalaKoidz } \\
\text { Saposhnikovia Divaricata (Turcz) } \\
\text { Schischk }\end{array}$ & $\begin{array}{l}\text { Lung and Spleen } \\
\text { deficiencies }\end{array}$ & Clinic \\
\hline
\end{tabular}

Table 2 Periodic Herbal Managements from G Xia's experience. ${ }^{20,21,34,39,40}$

\begin{tabular}{lll}
\hline Periodic stage & Treating Principle & Xia's herbal management $^{39,44}$ \\
\hline & Cangzhu / Xanthium Sibiricum Part. \\
& Xiangfu / CyperusRotundus L. \\
& Mudanpi /PaeoniaSuffruticosaAndr. \\
& Danshen /Salviaemiltiorrhizae Bge \\
& Chishao / PaeoniaLactiflora Pall. \\
To move Qi's stagnation and activity & Wulingzhi \\
blood for supporting follicle's & Zelan / LycopusLucidusTurcz var. \\
growth. & Hirtus Regel \\
& Zishiying / \\
& Shanzha / CrataegusPinnatifidaBge \\
& Yimucao / LeonurusJaponicus Hoult \\
& Fuling / Poria Cocos (Schw.) Wolf
\end{tabular}


Table Continued..

\begin{tabular}{|c|c|c|}
\hline Periodic stage & Treating Principle & Xia's herbal management ${ }^{39,44}$ \\
\hline Post of Menstruation & $\begin{array}{l}\text { To nourish Yin and blood for } \\
\text { promoting essence (egg)'s growing } \\
\text { up and bursting out }\end{array}$ & $\begin{array}{l}\text { Danggui / Angelica Sinensis (Oliv.)Diels } \\
\text { Chishao / PaeoniaLactiflora Pall } \\
\text { Shudihuang / RadixGlutinosaLibosch } \\
\text { Mudanpi /PaeoniaSuffruticosaAndr } \\
\text { Fuling / Poria Cocos (Schw.)Wolf } \\
\text { Shanyao / DioscoreaOppositaThunb } \\
\text { Shanyurou / Cornus Officinalis Sieb et. } \\
\text { Zucc } \\
\text { Zhizi / Gardenia Jasminoides Ellis } \\
\text { Chuanduan / DipsacusAsperoides C.Y. } \\
\text { Cheng et } \\
\text { Tusizi / CuscutaGlabraRoxb } \\
\text { Fupenzi / RubusChingi Hu }\end{array}$ \\
\hline Ovulation & $\begin{array}{l}\text { To tonify Kidney Yang \& to nourish } \\
\text { Yin essence and blood for preparing } \\
\text { a pregnancy }\end{array}$ & $\begin{array}{l}\text { Danggui / Angelica Sinensis (Oliv.)Diels } \\
\text { Danshen / Salviaemiltiorrhizae Bge } \\
\text { Chishao / PaeoniaLactiflora Pall. } \\
\text { Zelan / LycopusLucidusTurcz var. } \\
\text { gHirtus Regel } \\
\text { Chongweizi / LeonurusHeterophyllus } \\
\text { Sweet } \\
\text { Honghua / CarpesiumTinctorins L. } \\
\text { Xiangfu / CyperusRotundus L }\end{array}$ \\
\hline Before of Menstruation & $\begin{array}{l}\text { To move Liver Qi stagnation \& } \\
\text { nourish blood for feeding embryo } \\
\text { and preventing a miscarriage }\end{array}$ & $\begin{array}{l}\text { Chaihu / BupleurumChinense DC } \\
\text { Xiangfu / CyperusRotundus L } \\
\text { Aiye / Artemisisia Argyi Levl.et Vant } \\
\text { Xuduan / Dipsacus Asperoides C.Y. } \\
\text { Cheng et } \\
\text { Duzhong / EucommiaUlmoidesOliv. } \\
\text { Ejiao / AsiniGelatinum, ComiAsiniColla } \\
\text { Sangjisheng / TaxillusChinensis (DC.) Danser }\end{array}$ \\
\hline
\end{tabular}

However a review published by J Zhang. ${ }^{35}$ indicated a different opinion that there is limited evidence that the addition of CHM to clomiphene is associated with improved clinical pregnancy outcomes, and found no evidence of any other effect. ${ }^{35}$

To treat ovulation failure and insufficiency of stored ova: J Zhou's team observed the curative effect of CHM of Tonifying the Kidney and Activating the Blood on improving ovulation failure, and the effect on incidence rate of luteinized unruptured follicle (LUF) and luteal phase defect (LPD). 52 patients with ovulation disorder were randomly divided into two groups; one group was treated with traditional Chinese medicine ( $\mathrm{n}=32$, Table 1), and the other group, as the control, was treated with Western drugs $(n=20)$. In the treatment group, the patients were given various herbal formulae according to the patients' stages of the menstrual circle; the Western drug control group patients were given clomiphene $50-100 \mathrm{mg}$ per day on day 5 of menstrual or withdrawal of flavolutan. The two groups were all treated for three to six cycles. All 52 patients completed the treatment. The authors found that during the 156 cycles of 32 patients in the TCM group, ovulation was found in 133 cycles, and the ovulation rate was significantly higher than in the Western drug control group $(85.26 \%$ versus $58.7 \%$, $\mathrm{p}<0.05$ ). They found that the occurrence rates of LUF and LPD in the TCM group were lower than in the Western drug control group. The authors concluded that CHM, by the Tonifying kidney and Activating Blood method improved ovulation rate and lowered incidence rate of LUF and LPD when compared with clomiphene. ${ }^{36}$ (Table 2).

Periodic treatment method of CHM is a particular TCM technique using various herbal formulae in different stages of the menstrual circle to promote ovarian function and following the cyclical hormonal changes. It has been observed to be more effective than the standard TCM treatment methods. This method was created by Prof G Xia, ${ }^{39}$ and was developed by D Jiang into a regime which is suitable to be used in the West. ${ }^{37}$ (Table 2).

To treat Fallopian tube obstruction: J Kang's team studied an effective and practical treatment to Fallopian tube obstruction $(\mathrm{OvO})$. 120 patients with OVO were randomly divided into three groups: the TCM-WM group, treated with integrative Chinese herbal medicine (an established CHM formula) and Western medicine (salpingectomy); the TCM group, treated with Chinese herbal medicine alone; and the WM group treated with Western medicine alone. The therapeutic effect as well as the effect of treatment on serum C-reactive protein (CRP) and interleukin-1 beta (IL-1 beta) were observed. The authors found that after treatment, Fallopian tube patency rate rose to $86.7 \%$ and pregnancy rate to $85.0 \%$ in the TCM-WM group, while in the TCM group rates were $66.7 \%$ and $63.3 \%$ respectively, and in the WM group $53.3 \%$ and $50.0 \%$ respectively. Comparison among the three groups show that the effect in the TCM-WM group was significantly superior to that in the other two groups $(\mathrm{P}<0.01)$. The levels of CRP and IL-1 beta were all lowered after three courses of treatment, and the effect was more evident in the TCM-WM group $(\mathrm{P}<0.01)$. They authors concluded that TCM-WM treatment was a good and practical method in treating Fallopian tube obstruction. ${ }^{38}$

Y Jiang's team also compared the effect of treatment of Fallopian tube obstructive infertility by salpingectomy alone and in combination with TCM drugs for BushenHuoxue (invigorating Shen and promoting blood circulation, an established CHM formula, Table 1). To all the patients, salpingostomy was performed three to seven days after menstruation, and hydrotubation with Xiangdan Injection (XI) was applied once in the next menstrual cycle. BushenHuoxue Decoction (BHD) was administrated additionally to patients in the treatment group, one dose every day starting from the fifth day of 
menstrual cycle for 14 days. Three months' treatment was taken as one therapeutic course, and the observation lasted for four courses. The authors confirmed the combined therapy of salpingostomy and CHM was an effective therapy for Fallopian tube obstructive infertility by enhancing follicular development and increasing thickness of endometrium, and could elevate the pregnancy rate in patients. ${ }^{39}$

To treat PCO $\square$ PCOS: F Lian 's team found that CHM-ErzhiTiangui Granule (Table 1) could increase anti-Müllerian hormone (AMH) and oocyte quality for patients with polycystic ovarian syndrome. The drug's mechanisms of action are correlated with regulating $\mathrm{AMH}$ levels in the serum and follicular fluid, adjusting androgen levels, improving the pathophysiological changes of PCOS patients, and activating the ovarian microenvironment. ${ }^{40}$

C Lu' team divided 30 patients with PCOS into a normoinsulinaemic group $(\mathrm{NI}=13)$, and an hyperinsulinaemic group $(\mathrm{HI}=17)$. Both groups were treated for three menstrual cycles with the TCM Formula BushenHuatan Formula (an established CHMformula for tonifying kidney and activating blood, Table1). A metabolomics-approach based on ultra-high-performance liquid chromatography (UPLC) coupled with linear ion trap Orbi-trap mass spectrometer (LTQ Orbi-trap MS) was used to investigate serum metabolic changes of TCM intervention to PCOS. After BHF intervention for three menstrual cycles, the serum levels of glycerophosphoryl ethanolamine (GPEA), creatine, and creatinine decreased in both NI and HI groups. Furthermore in the NI group, the main manifestation were changes in phospholipid metabolism. While in the HI group, lysine, phenol sulfate, phe-phe etc. were decreased, and ornithine, proline, betaine, acetylcholine etc. were increased. Combined with clinical biochemical data, BHF was proved effective in treatment of PCOS by reducing the inflammatory reaction and oxidative stress. This study also illustrated that the LCMS-based metabolomics approach was a helpful tool to evaluate curative effect and to understand the mechanisms of TCM. ${ }^{41}$

To reduce and to treat miscarriage: Recurrent spontaneous miscarriage (RSM) is one of the commonest and complicated medical conditions amongst pregnant women. In recent years miscarriage rates have been progressively increasing, due to women trying to conceive later in life and the higher risk of pregnancy loss from in vitro fertilisation (IVF) and intrauterine insemination (IUI). TCM is often able to treat and prevent RSM.

Y Chen's team observed the effect produced by the Gushenantai recipe (a classical formula to prevent and treat miscarriage, Table 1) to women with recurrent spontaneous miscarriage without obvious cause, and its influence on T-lymphocytes. 90 patients were separated into two groups where routine treatment of Progesterone was given, and Gushenantai recipe given to the half in the treatment group. No herbs were given to the other half in the control group. The authors found that miscarriage was prevented and pregnancy proceeded in $71.1 \%$ in the control group, and $86.7 \%$ in the treatment group. The team also observed that B-HCG, HPL. P, CD4, CD8, CD4/CD8 levels were different before and after treatment. They were able to confirm that Gushenantai recipe was effective in reducing recurrent spontaneous miscarriage, and could correct immunological disorders and increase hormone levels. ${ }^{42}$

To treat mycoplasma infection: $\mathrm{Y}$ Lou's team investigated the changes in cytokines (IL-1beta, IL-2, TNF-alpha) in the peripheral blood and cervical mucus of infertile women with mycoplasma infection and the effect of intervention of TCM. 72 patients with positive genital mycoplasma cultures were randomly divided into a TCM group (38 cases) and a Western medicine group (34 cases). The Western medicine group was treated with $0.5 \mathrm{~g}$ azithromycin for three days and consecutively underwent six courses of treatment, each course of treatment of four days' duration. The TCM group was treated with Xiaozhi decoction (an established CHM formula) twice every day for six weeks. Cytokines (IL-1beta, IL-2, TNF-alpha) in the peripheral blood and cervical mucus were found to increase, suggesting that TCM could effectively inhibit the levels of IL-1beta, IL-2, TNF-alpha in the peripheral blood, and IL-1beta, TNF-alpha in cervical mucus. The authors concluded that Xiaozhi decoction could be used to treat infertile women with mycoplasma infection. ${ }^{43}$

To re-regulate immunology disorder: $\mathrm{J}$ Yu studied the effect of Yupingfeng Formula (a classic CHM formula)to treat infertility in women with immunological disorders. Table 1). 72 patients with immunological infertility were randomly assigned into two groups of 36. The observed group was given Yupingfeng granule; the control group was given oral Prednisone. Levels of AsAb, AEmAb, AOVAb and $\mathrm{AbCGAb}$ were markedly more reduced in the observed group than in the controlled group $(\mathrm{p}<0.05)$. Yu concluded that Yupingfeng granule was able to significantly affect immunological infertility. ${ }^{44}$

To treat many abnormal physical and psychiatric symptoms, Such as anxiety, stress, and insomnia: $\mathrm{K}$ Ried et al. ${ }^{28}$ designed questionnaires and interviewed women with infertility with the aim of identifying their quality of life. They found high level of distress, guilt, grief, and frustration caused by infertility. However, these women represented a highly motivated sample, who were actively seeking alternative support methods. Traditional Chinese Medicine addresses some of these. The group recommended that more peerled and professional-led support groups were greatly needed for women experiencing infertility to help break isolation and raise awareness of integrative approaches to fertility managemen. ${ }^{45}$

\section{Chinese Herbal Medicine can increase the success rate of in-vitro fertilisation and embryo transfer (IVF- ET) outcomes}

A review series by $\mathrm{J}$ Liu et al. found that CHM could increase success rates from $33 \%$ to $60 \%$ in IVF and IVFET treatment. The authors' results are summarised below: Twenty trials involving 1721 women were included in the metaanalysis. Three trials were evaluated as having an unclear risk of bias. The remaining trials were evaluated as having a high risk of bias. Despite this the authors found that the combination of CHM and IVF significantly increased clinical pregnancy rates (OR 2.04, 95\% CI 1.67 to $2.49, \mathrm{p}<0.00001$ ), and ongoing pregnancy rates (OR $1.91,95 \% \mathrm{CI}$ 1.17 to $3.10, p=0.009$ ). Use of CHM after embryo transfer had no better outcome in reducing the rate of ovarian hyperstimulation syndrome (OR $0.39,95 \% \mathrm{CI} 0.14$ to $1.11, \mathrm{p}=0.08$ ). This meta-analysis showed that combination of IVF and CHM used in the included trials improve IVF success, however due to the high risk of bias observed with the trials, the significant differences found were felt to be unlikely to be accurate. The authors also mentioned that no conclusion could be made with respect to the reproductive toxicity of CHM. Further large randomized placebo controlled trials were recommended to confirm these findings before recommending women to take CHM to improve their IVF success. ${ }^{47}$

F Lian's team conducted a series of studies to confirm the effectiveness of CHM in promoting IVF and increasing pregnancy rates:

To promote pregnancy uterus receptivity: Lian et al.. ${ }^{48}$ found that for infertile patients undergoing IVF, the recipe for tonifying the Kidney (Table 1) as an adjunct to standard Western treatment could reduce Gn dosage and treatment duration, alleviate clinical symptoms, and 
improve pregnancy rate. The authors felt that the improvement could be explained by an increased level of DNMT1 protein expression after treatment, leading to enhanced endometrial receptivity. ${ }^{48}$

To improve the quality of oocytes and leukemia inhibitory factor in follicular fluid: The established CHM formula Erzhitiangui Granule ETG (Table 1) was found to distinctively increase the amount of oocytes, elevate the quality of embryos, and raise the success rate of IVF-ET. The authors speculated that the mechanism maybe correlated to the increase of LIF level in FF and the activating of microenvironment for its full expression. ${ }^{49}$

To improve endometrial blood flow in the uterus: Endometrial blood flow is directly related to endometrial receptivity, thereby affecting in-vitro fertilization and embryo transfer (IVF-ET) outcomes. J Guo team's studies confirmed that CHM formulae (Table 1) based on reinforcing kidney and activating blood can promote the formation of uterine endometrial blood vessels by adjusting expression of a variety of angiogenic growth factors, and regulating nitric oxide levels for the inhibition of vascular smooth muscle contraction of the uterus. Treatments based on differentiation of syndromes are key to the theory of TCM. Differentiation of syndromes should be combined with biomedical disease diagnosis. The authors also felt that it was necessary to further clarify other endometrial blood flow disorders using TCM diagnostic methods. ${ }^{50}$

To decrease FSH level for making a good preparation to IVF treatment: Elliott reported that Follicle stimulating hormone (FSH) levels are routinely tested during biomedical investigations into female fertility. An appropriately low FSH level is frequently required by fertility clinics as an entry requirement for women wishing to receive assisted reproductive technology (ART) treatment such as invitro fertilisation (IVF). The author asserts that with the appropriate treatment using acupuncture and Chinese herbal medicine, the proper functioning of the ovaries and anterior pituitary gland, in cases where they have been pathologically affected, can be restored. This process does not, however, necessarily imply that treatment has reversed the ageing process or affected the quality of eggs produced. ${ }^{49}$

To support patients who have failed IVF treatment: X X's team explored the effect of traditional Chinese comprehensive therapy (TCCT) on promoting gestation in patients with previously failed invitro fertilisation and embryo transfer (IVF-ET) because of kidney deficiency, liver stagnation, and blood stasis (KLB). 37 patients were enrolled in this study and divided into two groups: a trial group with 35 patients, and a control group with 32 patients. The trial group was given TCCT for three months, then administered IVF-ET or natural pregnancy was awaited. The control group was administered IVFET without TCCT three months after the previous IVF-ET or natural pregnancy attempt. The patterns of KLB were observed both before and after treatment. The natural pregnancy rate of the two groups was calculated after treatment.

After treatment with TCM comprehensive therapy, seven patients in the treatment group became pregnant, while there were no successful conceptions in the control group. The difference in clinical pregnancy rate in the initial cycle and transfer cycle of IVF were significantly different $(p<0.05)$. The trial group had a significantly higher conception rate than the control group $(\mathrm{P}<0.05)$. The authors concluded that TCCT can promote natural pregnancy rate in patients with previously failed IVF-ET. TCCT could increase the number of fertilised eggs, the fertilisation rate, pregnancy rate, and clinical pregnancy rate after another IVF-ET treatment. ${ }^{50}$

\section{Whole Systematic TCM Treatment for Infertility and IVF Treatment}

TCM is a traditional medical system with a thousand years of experience which should be combined with herbal medicine and acupuncture together. From the evidence it appears that using whole TCM treatment may achieve the best results for both natural pregnancy and IVF.

K Sela et al.. ${ }^{51}$ observed the effect of combining acupuncture and medicinal herbs as a therapeutic adjuvant to ovulation induction with intrauterine insemination (IUI) procedures, and evaluated its contribution to pregnancy and "take-home baby" rates. The retrospective study was carried out in a university - affiliated municipal hospital. All women undergoing artificial insemination by donor spermatozoa (AID) and concomitantly treated with TCM were invited to participate. The enrolled women underwent weekly TCM in parallel with standard Western therapy. The treatment lasted between two and 36 cycles (equivalent to a time period ranging from one month to one year). The control group was comprised of women who underwent AID without TCM and whose data were retrospectively retrieved from hospital files. Pregnancy was assessed by human chorionic gonadotropin findings in blood 12-14 days after IUI. The birth rate was calculated during follow-up. A total of 29 women aged 30-45 years were enrolled in the study. The historical control group include 94 women aged 28-46 years. The results showed that women who combined TCM with procedures for undergoing IUI had significantly higher pregnancy $(\mathrm{OR}=4.403$, 95\% CI 1.51-12.835, $\mathrm{p}=0.007)$ and birth rates $(\mathrm{OR}=3.905,95 \%$ CI $1.321-11.549, \mathrm{p}=0.014)$ than the control group. The group concluded that TCM appeared to be beneficial as an adjunctive treatment in IUI procedures. They called for randomised controlled trials to further assess the role of acupuncture and herbs in this setting..$^{51}$

E Lee et al. ${ }^{52}$ observed that whole-systems traditional Chinese medicine (WS-TCM) was a multi-dimensional intervention that could include any combination of modalities classified under the system of TCM. They describe these as including any combination of acupuncture (the insertion of sterile, filiform needles in the body), moxibustion (the burning of the processed herb Artemesia argyi on or near the body), and Chinese herbal medicine (granule, powder or dry herbal forms). The authors compared the reproductive outcomes of women who elected for WS-TCM treatment in addition to their usual IVF care, and compared them with those who received the usual IVF care alone, and to those who received two standardised acupuncture treatments on the day of embryo transfer acupuncture only. They compared the three groups on the outcome of live birth, and found evidence that the addition of WS-TCM to non-donor IVF cycles increased the odds of achieving a live birth over usual IVF care alone or two standardized treatments administered around embryo transfer. They found that the effects of WS-TCM were less clear compared with usual donor IVF care alone but showed a nonsignificant trend towards increased odds of a live birth in this cycle type. This retrospective cohort study suggested that WS-TCM as an adjuvant IVF treatment may be associated with improved live birth rates. $^{52}$

\section{Conclusion}

From searching and reviewing the above information, some conclusions can be drawn: 
i. Chinese Herbal medicine is an effective therapy which appears to be show ability to support infertility treatment. Much research has been done in China to explain the mechanism of action of CHM in treating Infertility, and some utilise very modern techniques and high levels of expertise. ${ }^{21-27}$ However they are limited in circulation and impact due to being in Chinese only.

ii. A mature and established CHM formula may be the key to high efficacy, and effective and experienced herbal formulae should be designed according to diagnostic patterns within TCM..$^{53,54}$ No paper described effects produced by a single herb to treat Infertility.

iii. Combining the whole system of TCM together, such as combining acupuncture and CHM, acupuncture, moxibustion and CHM, massage and CHM, gives better treatment power than individual therapy modalities. ${ }^{55,56}$

iv. The shortcoming of CHM may still be its weaker treatment level. Some researchers observe that combining CHM with Western medical technology or drugs can give better effects than either CHM or Western drug alone. . $^{38,39,42}$

\section{Acknowledgments}

None.

\section{Conflicts of Interset}

None.

\section{References}

1. Clinic Specialization Gynaecology handbook Oxford University publisher.

2. Dunson DB, Baird DD, Colombo B Increased infertility with age in men and women. Obstet Gynecol. 2004;103(1):51-56.

3. National Centre for health statistics: Infertility.

4. Wikipedia, Infertility Females.

5. Richard S, Unexplained Infertility Background, Tests and Treatment Options. Advanced Fertility Centre of Chicago, USA.

6. Williams T, Mortada R, Porter S Diagnosis and Treatment of Polycystic Ovary Syndrome. Am Fam Physician. 2016; 94(2):106-113.

7. Sotrel G Is Surgical Repair of the Fallopian Tubes Ever Appropriate? Rev Obstet Gynecol. 2009; 2(3):176-185.

8. Lang EK, Dunaway HE Jr, Roniger WE Selective ostealsalpingography and transvaginal catheter dilatation in the diagnosis and treatment of fallopian tube obstruction. AJR Am J Roentgenol. 1990;154(4):735-740.

9. Pandian Z, Gibreel A, Bhattacharya S In vitro fertilisation for unexplained subfertility. Cochrane Database Syst Rev. 2015;(4):CD003357.

10. Chambers GM, Wand H, Macaldowie A, et al. Population trends and live birth rates associated with common ART treatment strategies. Hum Reprod. 2016;31(11):2632-2641.

11. Rashidi M, Aaleyasin A, Aghahosseini M, et al. Advantages of Recombinant Follicle-Stimulating Hormone over Human Menopausal Gonadotropin in Intrauterine Insemination: A Randomized Clinical Trial in Polycystic Ovary Syndrome-Associated Infertility. Eur J Obstet Gynecol Reprod Bio. 2015;1169(2):244-247.

12. Freeman MP, Toth TL, Cohen LS, et al. Assisted reproduction and risk of depressive relapse: considerations for treatment. Annals of Clinical Psychiatry. 2013;25 (4):283-288.
13. F Qu, D Jiang, Mark bovey, Giovanna F, Kelvin C, Carolina S, et al. Does acupuncture improve the outcome of in vitro fertilization? Guidance for future trials. European Journal of Integrative Medicine. 2012 4(3):234-244.

14. Chang, Chung PH, Rosenwaks Z Role of acupuncture in the treatment of female infertility. i. 2002;78(6): 1149-1153.

15. Cavalla, Hilida Acupuncture and Electroauriculo therapy Treatment for Infertility, Medical Acupuncture. 2010; 22(1):49-52.

16. Zhou J, Qu F Treating gynaecological disorders with traditional Chinese medicine: a review. Afr J Tradit Complement Altern Med. 2009; 6(4): 494-517.

17. Xu X, Hang Yin, Daiyi T, Li Z, Roger G Application of traditional Chinese medicine in the treatment of infertility. Human Fertility (Camb). 2003; 6 (4):161-168.

18. Xia JF, Inagaki Y, Zhang JF, Wang L, Song PP Chinese medicine as complementary therapy for female infertility. Chin J Integr Med. 2016; 23(4): 245-252.

19. Park JJ, Kang M, Shin S, Choi E, Kwon S, et al. Unexplained infertility treated with acupuncture and.2010 20102010herbal medicine in Korea. J Altern Complement Med 16(2): 193-198.

20. Hung YC, Kao CW, Lin CC, Liao YN, Wu BY, et al Chinese Herbal Products for Female Infertility in Taiwan: A Population-Based Cohort Study. Medicine (Baltimore. 2016; 95(11): e3075.

21. Yu Nan, Yang Jing, Yin T Extracts from a traditional Chinese herbal remedy (Zhuyun recipe) improve endometrial receptivity in mice with embryonic implantation dysfunction and ovulation stimulation. $J$ Ethnopharmacol. 2011; 137(1): 389-395.

22. X Gong, Lou J, Lu Q, Huang H, Jin Z Bu Shen HuoXue decoction restores endometrial leukemia-inhibitory factor but not Angiopoietin-2 expression, and improves uterine receptivity in the controlled ovarian stimulation rat model. Exp Ther Med. 2015; 9(3):751-756.

23. S Li, S Luo Study on Kidney Tonifying of CHM to the Rat Models with Ovulated inhibition, Chinese Journal of Basic Medicine in TCM. 2010; $16(7): 567-569$.

24. X Teng, et al. Study on mechanism of regulation and control of Yulin formula of CHM to hormone level and histomorphology of SD rat model of premature ovary failure. Assay of Master degree of TCM gynaecology, BUCM, China. 2016

25. S Luo Study of Mechanism on Zhuyun No3 in preventing and treating spontaneous abortion. Chinese Journal of integrated traditional and western medicine. 2003; 23(7):522-525.

26. H Zhao, S Luo, et al. Effects of Zhuyun No3 Serum TG-B1 in Abortion Rats of Kidney Deficiency and Luteum Inhibition Shanghai. Journal of TCM. 2008; 42(12):71-73.

27. H Du, Chang X, Du H, Zhang M, Zhang J, et al. Effect of soothing liver therapy on oocyte quality and growth differentiation factor- 9 in patients undergoing in vitro fertilization and embryo transfer. J Tradit Chin Med. 2013; 33(5):597-602.

28. K Ried, Stuart K Efficacy of Traditional Chinese Herbal Medicine in the management of female infertility: a systematic review. Complement Ther Med. 2011;19(6):319-331.

29. Ried K, Ann A Quality of life, coping strategies and support needs of women seeking Traditional Chinese Medicine for infertility and viable pregnancy in Australia: a mixed methods approach BMC Women's Health. 2013;13:17-19.

30. Ried K Chinese herbal medicine for female infertility: an updated metaanalysis. Complement Ther Med. 2015; 23(1):116-128. 
31. Tan L, Tong Y, Sze SC, Xu M, Shi Y, et al. Chinese herbal medicine for infertility with anovulation: a systematic review. J Altern Complement Med. 2012;18(12):1087-1100.

32. Q Chen Effect of Tiaojing Yunyu recipe on follicular and endometrial growth, and blood flow characteristics by simultaneous testing in patients with anovulatory infertility. Chinese Journal on Research and Formula. 2004;0(2):58-61.

33. X Li Clinical study on traditional Chinese medicine in improving endometrial receptivity during ovarian stimulation, Contemporary Diagnosis and Treatment. 2009; 20(3):147-149.

34. Y Huang, Cheng Q, Zhou AF Clinical observation on anovulatory infertility of blood and essence asthenia type treated by Lin'erlai Prescription. Zhongguo Zhong Xi Yi Jie He Za Zhi. 2007;27(11):10281030.

35. Zhou K, Zhang J, Xu L, Wu T, Lim CE Chinese herbal medicine for subfertile women with polycystic ovarian syndrome. Cochrane Database Syst Rev. 210; 10: CD007535.

36. J Zhou, Li Da-jian Tonifying kidney and activating blood method in treating ovulation failure of 32 cases: B ultrasonic evaluation. Chinese Journal of Clinical Rehabilitation. 2006;10(31):114-116.

37. D Jiang Treating Female Infertility by Strengthening the Natural Menstrual Cycle with Acupuncture and Chinese Herbal Medicine. The European Journal of Oriental Medicin. 2014; e 7(6):28-35.

38. J Kang, Xia W, He QY Clinical studies on treatment of oviduct obstruction by integrative traditional Chinese and Western medicine. Zhongguo Zhong Xi Yi Jie He Za Zhi. 2001; 21(6): -418.

39. Y Jiang, Jiang YC, Liang RN, Liu RT Effects of combined therapy of salpingostomy and BushenHuoxue drugs on fallopian tube obstructive infertility. Zhongguo Zhong Xi Yi Jie He Za Zhi. 2006; 26(4):325-328.

40. F Lian, Zhao S Effects of Shen invigorating and Chong-channel regulating method on anti-Müllerian hormone and oocyte quality in polycystic ovarian syndrome patients. Zhongguo Zhong Xi Yi Jie He Za Zh. 2012;i 32(1): 9-12.

41. C Lu, Zhao X, Li Y, Li Y, Yuan C, et al. Serum metabolomics study of Traditional Chinese medicine formula intervention to polycystic ovary syndrome. J Pharm Biomed Anal. 2016; 20(120): 127-133.

42. Y Chen Clinical observation to Gushenantai Recipe treating Recurren spontaneous miscarriage Clinical study 1.2016

43. Y Lou, Yang XF, Liu LC Study on changes in cytokines of infertile women with mycoplasma infection and intervention with traditional Chinese medicines. Zhongguo Zhong Yao Za Zhi. 2012; 37(20): 31583160 .
44. J Yu Observation to Yupingfeng Granule treating Immunological infertility. China Modern Doctor.2010; 48(12).

45. H Cao, Han M, Ng EH, Wu X, Flower A, et al. Can Chinese herbal medicine improves outcomes of in vitro fertilization? A systematic review and meta-analysis of randomized controlled trials. PLoS One. 2013; 8(12): e81650.

46. F Lian, Rui-Xia W, Feng-Mei M, Zhen-Gao S, Li-Hong W, et al. Effects of Chinese medicines for tonifying the kidney on DNMT1 protein expression in endometrium of infertile women during implantation period. J Altern Complement Med. 2013; 19(4):353-359.

47. F Lian Clinical study on effect of ErzhiTiangui Granule in improving the quality of oocytes and leukemia inhibitory factor in follicular fluid of women undergoing in vitro fertilization and embryo transfer. Chinese Journal of Integrated traditional and western Medicine. 2007

48. J Guo, Wang LN, Li D Exploring the effects of Chinese medicine in improving uterine endometrial blood flow for increasing the successful rate of in vitro fertilization and embryo transfer. Zhong Xi Yi Jie He Xue Bao. 2011; 9(12):1301-1306.

49. D Elliott The treatment of elevated FSH levels with Chinese medicine. Journal of Chinese Medicine. 2009; (91): 5-7.

50. XX, Weining J, Shuhua C, Yanmei Z, Wanting X, et al. Effect of traditional Chinese comprehensive therapy on gestation in patients with previously failed in vitro fertilization and embryo transfer. $J$ Tradit Chin Med. 2015; 35(4):428-433.

51. K Sela, Ofer Lehavi, Amnon B, Karin Kedar S, Halim Y, et al. Acupuncture and Chinese herbal treatment for women undergoing intrauterine insemination. European Journal of Integrative Medicine. 2011; 3(2): 77-81

52. E Lee, Opsahl MS, Wiemer KE, Mist SD, Caughey AB Impact of Whole Systems Traditional Chinese Medicine on In Vitro Fertilization Outcomes. Reprod Biomed Online. 2015; 30(6):602-612.

53. Birkeflet Poor multi-rater reliability in TCM pattern diagnoses and variation in the use of symptoms to obtain a diagnosis. Acupunct Med. $2014 ; 32(4): 25-328$

54. M Coyle A survey comparing TCM diagnosis, health status and medical diagnosis in women undergoing assisted reproduction. Acupunct Med. $2005 ; 23(2): 62-69$.

55. G Xia Female and Men infertility and regulation in menstrual circles. People Health Publishing House, Beijing, The secondary publishing issue. 2001

56. Z Dong The Experienced formulae of TCM on Infertility Beijing. Publishing House of Science and Technology Beijing. 1990. 